\title{
Utilização de planejamento em rede Simplex na previsão de propriedades mecânicas de peças cerâmicas vermelhas
}

\section{(The use of Simplex design to predict the mechanical properties of brick ceramic)}

\author{
J. Alexandre, R. Toledo, F. Saboya, M. Pereira \\ UENF Centro de Ciência e Tecnologia - CCT \\ Universidade Estadual do Norte Fluminense \\ Av. Alberto Lamego, 2000, Horto, Campos dos Goytacazes, RJ, Brasil, 28015-620 \\ saboya@uenf.br
}

\begin{abstract}
Resumo
As indústrias cerâmicas de um modo geral, no seu processo produtivo, utilizam matérias-primas provenientes de sedimentos argilosos e/ou alterações de minerais formadores de rochas. Trata-se portanto de um material com alto grau de heterogeneidade tanto do ponto de vista mineralógico quanto em relação ao grau de alteração dos seus minerais, dificultando sobremaneira a previsão do comportamento mecânico do produto final (tijolos, telhas, etc.). Uma prática que é comumente adotada durante a confecção de peças cerâmicas é a mistura de diferentes tipos de solo, realizada muitas vezes, de maneira intuitiva e empírica, onde as características do produto são fortemente dependentes das proporções usadas nessas misturas. O presente trabalho apresenta uma metodologia numérica, utilizando o planejamento em rede Simplex, na previsão das propriedades resultantes destas misturas. Dependendo do modelo escolhido (linear, quadrático, cúbico, etc.), o resultado de uma mistura genérica de até três componentes pode ser determinado numericamente a partir do conhecimento prévio das propriedades de cada componente original, bem como das misturas em determinadas proporções fundamentais. Estes resultados foram testados empiricamente em escala laboratorial e podem ser adaptados posteriormente ao processo produtivo, possibilitando a economia de matéria-prima e reduzindo a exploração predatória das jazidas.
\end{abstract}

Palavras-chave: misturas, rede Simplex, cerâmica vermelha.

\section{INTRODUÇÃO}

As cerâmicas estruturais em geral utilizam como matéria-prima para confecção de seus artefatos, materiais argilosos provenientes principalmente do intemperismo das rochas e de depósitos sedimentares. Após os processos de intemperismo e/ou sedimentação os minerais decompostos ou simplesmente desagregados passam a formar um aglomerado constituindo a jazida de material ar-

\begin{abstract}
In general, the brick ceramic industry uses clayey soils from sedimentation basin and/or from weathered rock as raw material. Due to their nature, these soils, most of the time, are extremely heterogeneous at their natural state. This heterogeneity is not only related to their mineralogical composition but also related to their grading and the degree of mineral weathering. Therefore, an important question arises: how to predict a desirable specific behavior of these materials with such a variable characteristic? It is very well known that ceramic workers apply an empirical procedure, combining different materials with known properties to manufacture these products. Thus, this paper presents a method to help in overcome this problem by applying a numerical routine which is used to predict specific final properties of different soil admixtures. It is also a helpful tool to determine the ideal admixture proportion that will show some required property, starting from the previously known behavior of the original components and some basic admixtures. The obtained results by carrying out laboratory tests in small ceramic bricks are compared to those predicted ones in order to verify the model suitability. This study is performed in a laboratory scale but it can be easily adapted to the industrial production process, enhancing the economy and preserving the environment from the predatory exploration of borrow areas.

Keywords: mixtures, Simplex lattice, clay.
\end{abstract}

giloso, que possui características próprias dependentes de sua constituição e das condições de sua formação. Devido à complexidade de formação dessa matéria-prima, as previsões que podem ser feitas antes do seu processamento tornam-se um grande desafio do ponto de vista mecânico. Prever o comportamento de artefatos cerâmicos baseando-se nas propriedades mineralógicas de seus constituintes pode vir a se constituir em uma importante ferramenta na busca da qualidade de peças cerâmicas vermelhas. Um méto- 
do iterativo é apresentado neste trabalho, onde o solos puros e suas misturas correspondentes são analisadas a partir de um número mínimo de experimentos através de um modelo numérico denominado planejamento em rede Simplex [1].

\section{METODOLOGIA}

A metodologia proposta neste trabalho baseia-se em um modelo numérico que permita programar as misturas a serem utilizadas na confecção de produtos cerâmicos. $\mathrm{O}$ método adotado é o de planejamento em rede Simplex, onde modelos são ajustados para representar as respostas sobre uma superfície. Objetiva-se então encontrar um modelo que descreva o comportamento das misturas com a menor margem de erro possível, quando comparadas com os resultados reais dos experimentos, levando-se em conta o número mínimo necessário de experimentos.

O primeiro passo seria postular um ou mais modelos para descrever as propriedades de interesse (tensão de ruptura, porosidade, etc.) em função da composição das misturas. Em seguida, fazer um planejamento experimental, especificando as composições a serem estudadas. Uma avaliação da eficiência dos modelos pode ser feita através de métodos estatísticos e comparação entre os resultados experimentais e os obtidos com o modelo numérico.

\section{EXPERIMENTOS}

Para exemplificar a utilização do método, foram usadas três amostras de solo argiloso obtido na planície de inundação do rio Paraíba do Sul na região de Campos dos Goytacazes. As jazidas desta planície são basicamente constituídas de materiais argilosos cujo principal argilomineral é a caulinita, porém traços de gibsita e ilita são por vezes observados. Essas três amostras foram nomeadas de $X_{1}, X_{2}$ e $X_{3}$ e suas distribuições são mostradas no espaço Simplex que corresponde a um triângulo equilátero (Fig. 1). A soma das porcentagens dessas amostras $(X=0,1 / \mathrm{m}, 2 / \mathrm{m}, \ldots$,$) deve aten-$ der a restrição da equação (A)

$$
X_{1}+X_{2}+X_{3}=1
$$

A distribuição simétrica das misturas dentro desse espaço é conhecida como rede (lattice), onde uma mistura pode ser um elemento puro ou interações binárias ou ternárias entre os mesmos,

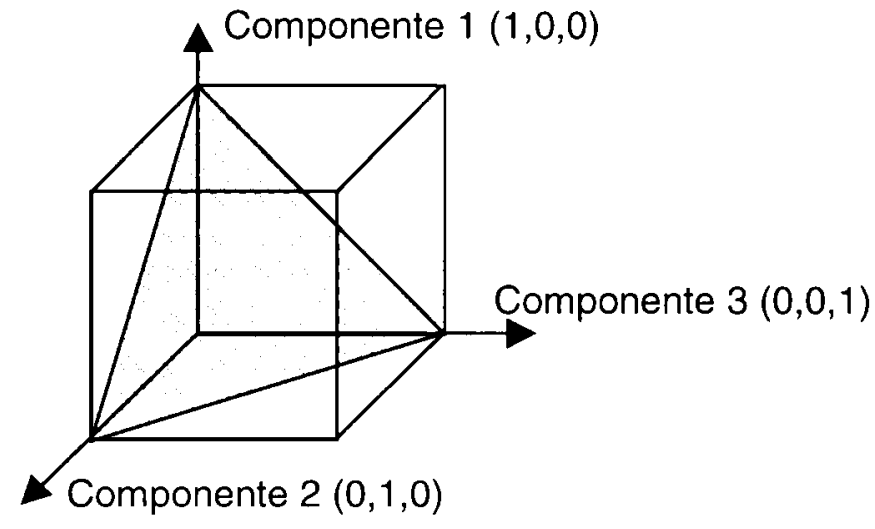

Figura 1: Espaço Simplex para três componentes.

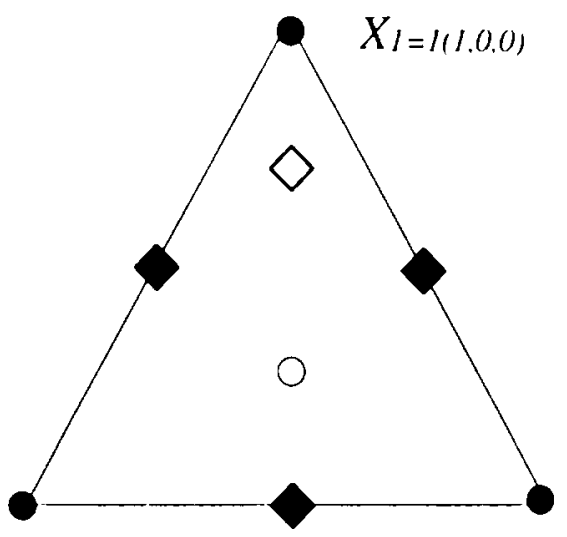

$$
X_{2}=1(0,1.0) \quad X_{3}=1(0.0 .1)
$$

$X_{\mathrm{i}}=1, \mathrm{X}_{\mathrm{j}}$ para $\mathrm{i}, \mathrm{j}=1,2,3 \mathrm{i} \neq \mathrm{j}$

$\diamond X_{1}>0, X_{2}>0$ e $X_{3}>0$

- $\mathrm{X}_{\mathrm{i}}=0, \mathrm{X}_{2}$ e $\mathrm{X}_{3} \neq 0$ ou $\mathrm{X}_{2}=0, \mathrm{X}_{1}$ e $\mathrm{X}_{3} \neq 0$ ou $\mathrm{X}_{3}=0, \mathrm{X}_{1}$ e $\mathrm{X}_{2} \neq 0$

- $X_{3}=1 / 3, X_{2}=1 / 3, X_{3}=1 / 3$

Figura 2: Distribuição de pontos em um espaço Simplex.

como mostrado na Fig. 2. Uma equação polinomial [2] de grau $m$, equação (B), pode ser usada para resolver o problema; isto é, para fazer uma análise de regressão que atenda à restrição da Equação (A) e passe a ter o número de termos igual ao número de pontos da rede Simplex (equação (C)). A partir da distribuição dos pontos na rede Simplex, atendendo à restrição da equação $(\mathrm{A})$, pode-se através da equação polinomial canônica (equação (B)), encontrar as respostas desejadas (propriedades) em função das misturas.

$\eta=\beta_{0}+\sum_{\mathrm{i}=1}^{\mathrm{q}} \beta_{\mathrm{i}} \mathrm{x}_{\mathrm{i}}+\sum \sum_{\mathrm{i} \leq \mathrm{j}}^{\mathrm{q}} \beta_{\mathrm{ij}} \mathrm{x}_{\mathrm{i}} \mathrm{x}_{\mathrm{j}}+\sum_{\mathrm{i}}^{\mathrm{q}} \sum_{\leq \mathrm{j}}^{\mathrm{q}} \sum_{\leq \mathrm{k}}^{\mathrm{q}} \beta_{\mathrm{ijk}} \mathrm{x}_{\mathrm{i}} \mathrm{x}_{\mathrm{j}} \mathrm{x}_{\mathrm{k}}+\ldots$

Na Equação B, polinomial de grau $m, \eta$ representa as respostas obtidas (observadas), $\beta$, os parâmetros do modelo e $x$ as proporções dos componentes na mistura.

$\eta=\sum_{\mathrm{i}=1}^{\mathrm{q}} \beta_{\mathrm{i}}^{*} \mathrm{x}_{\mathrm{i}}+\sum \sum_{\mathrm{i}<\mathrm{j}}^{\mathrm{q}} \beta_{\mathrm{ij}}^{*} \mathrm{x}_{\mathrm{i}} \mathrm{x}_{\mathrm{j}}$

O número de pontos em uma rede Simplex $\{q, m\}$ onde $q$ é o número de componentes e $m$ é o grau do polinômio é dado por $\frac{(q+m-1) !}{m !(q-1) !}$. O número de pontos em uma rede Simplex $\{q, m\}$ para $3 \leq q \leq 10,1 \leq m \leq 4$ onde o número de níveis para cada componente é $m+1$ é mostrado na Tabela I.

Apenas a título de exemplificar aplicações do método, foi feito um estudo da variável tensão de ruptura a partir de três amostras $\left(X_{1}, X_{2}, \mathrm{e} X_{3}\right)$ queimadas à temperatura de $1200^{\circ} \mathrm{C}$. Essas amostras foram moldadas e prensadas nas proporções estratégicas para que o grau dos modelos pudesse ser ajustado em função não apenas da aderência das respostas, mas também em função dos resultados de análise de erro. As interações entre as proporções amostradas são apresentadas na Fig. 3, onde o número de amostras para cada com- 
Tabela I - Número de experimentos em uma rede Simplex $\{q, m\}$ (apud [1]).

Grau do

Número de Componentes $q$

modelo

\begin{tabular}{ccccccccc}
$M$ & \multicolumn{1}{c}{ ( } & 4 & 5 & 6 & 7 & 8 & 9 & 10 \\
\hline 1 & 3 & 4 & 5 & 6 & 7 & 8 & 9 & 10 \\
\hline 2 & 6 & 10 & 15 & 21 & 28 & 36 & 45 & 55 \\
\hline 3 & 10 & 20 & 35 & 56 & 84 & 120 & 164 & 220 \\
\hline 4 & 15 & 35 & 70 & 126 & 210 & 330 & 495 & 715 \\
\hline
\end{tabular}

ponente foi ensaiado em triplicata para que inferências estatísticas pudessem ser feitas ao modelo utilizando-se para isso dos critérios t de Student e Fisher. As análises estatísticas não foram apresentadas neste trabalho, por necessitarem de uma abordagem mais específica; mas podem ser vistas em detalhes, assim como outros estudos para outras propriedades e temperaturas em [2].

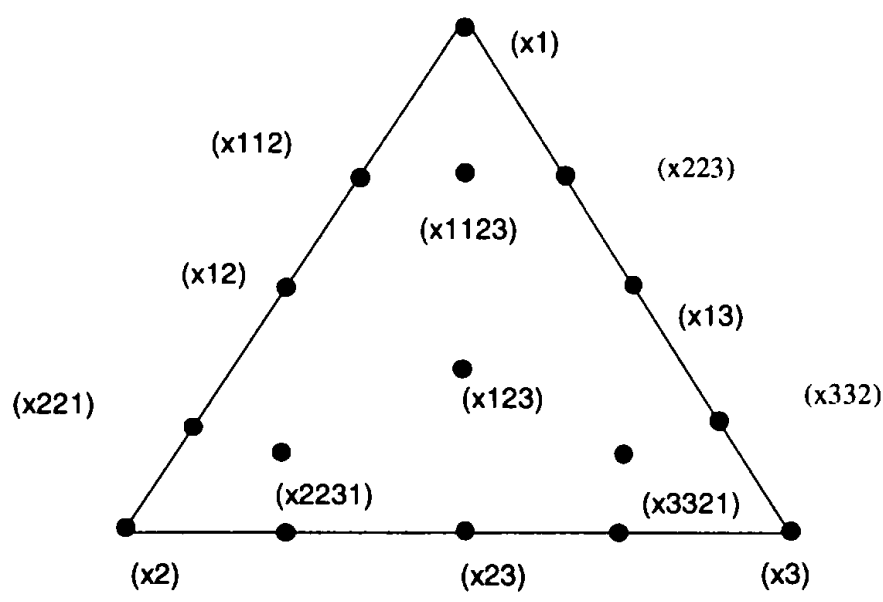

Figura 3: Distribuição de pontos que correspondem às misturas consideradas para a execução dos ensaios.

\section{PREPARAÇÃO DAS AMOSTRAS}

As amostras foram moldadas em forma de barras (Fig. 4) por prensagem, sob uma pressão de $25.0 \mathrm{MPa}$, com umidade de moldagem de 6,0\%. Após moldagem, as amostras foram secas em estufa e posteriormente queimadas em forno elétrico-resistivo

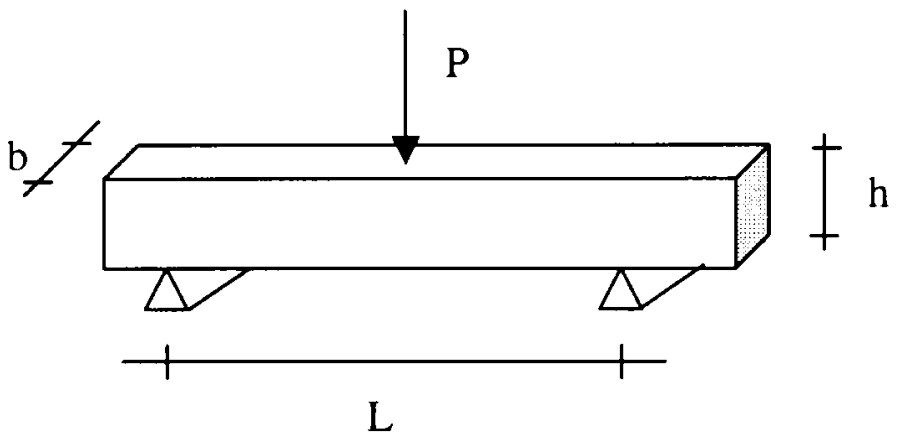

Figura 4: Aspectos típicos das amostras. a uma temperatura de $1200{ }^{\circ} \mathrm{C}$ sob taxa de aquecimento da ordem de $6{ }^{\circ} \mathrm{C} / \mathrm{min}$ e patamar de 3 horas. Após esta etapa, as amostras foram submetidas a ensaios de absorção de água, massa específica aparente, porosidade, variação linear e tensão de ruptura à flexão, onde neste trabalho só serão apresentados os resultados de tensão de ruptura à flexão, pois o principal objetivo é demonstrar a aplicabilidade do método.

$$
\mathrm{TR}=\frac{3 \mathrm{PL}}{2 \mathrm{bh}^{2}}
$$

$\mathrm{TR}$ - tensão de ruptura à flexão $(\mathrm{MPa})$

$\mathrm{P}$ - carga atingida no momento da ruptura $(\mathrm{kN})$

$\mathrm{L}$ - distância entre os apoios do corpo de prova (m)

$\mathrm{b}$ - largura do corpo de prova (m)

$\mathrm{h}$ - espessura do corpo de prova (m).

\section{DETERMINAÇÃO DOS MODELOS EM FUNÇÃO DAS RESPOSTAS OBTIDAS}

A partir dos resultados obtidos no experimento, uma avaliação estatística pode ser feita através da análise de variância (ANOVA) onde se determina um valor de $R^{2}$ que expressa o ajuste do modelo da fração descrita pela regressão, dada por:

$$
\mathrm{R}^{2}=\mathrm{SQ}_{\mathrm{R}} / \mathrm{SQ}_{\mathrm{T}}
$$

Onde $S Q_{R}$ e $S Q_{T}$ são a soma quadrática dos resíduos e dos erros totais, respectivamente.

O primeiro modelo postulado foi um modelo linear (Fig. 5a) onde a distribuição da rede Simplex é formada por três pontos localizados nos vértices do triângulo.

Após a avaliação, foi observado que os resultados não foram satisfatórios, em virtude do valor de $R^{2}$ ser da ordem de 0,62 , havendo a necessidade de aumentar o grau do modelo para um modelo quadrático (Fig. 5b). Este modelo é formado pelas três misturas puras que são os vértices do triângulo, mais três pontos sobre as arestas que são misturas binárias. Nestas, cada componente participa com $50 \%$ das proporções $\left(1 / 2\right.$ de $X_{1}, 1 / 2$ de $X_{2}$ e $1 / 2$ de $\left.X_{3}\right)$. Observandose que o modelo quadrático, apresenta uma melhoria considerável no valor de $R^{2}$ que foi da ordem de 0,93 , tentou-se um modelo mais complexo objetivando uma melhor aderência entre valores medidos e calculados. Considerou-se então um modelo cúbico especial (Fig. 5c), sendo composto por sete pontos, os seis do modelo de grau mais baixo e o sétimo formado por uma mistura ternária no centro do triângulo composta por $1 / 3$ de $X_{1}, 1 / 3$ de $X_{2}$ e $1 / 3$ de $X_{3}$ Obteve-se então um $R^{2}$ de 0,94 , que não se traduziu em uma melhoria significativa no resultado final quando comparado com o modelo quadrático.

O resultado obtido com os três modelos e a comparação feita entre estes e os resultados dos experimentos são mostrados na Tabela II. A Fig. 6 mostra a superfície de resposta para o modelo cúbico especial.

Os cálculos foram realizados com as seguintes equações $\hat{\mathrm{y}}_{\text {quadratico }}=182,87 \mathrm{x}_{1}+296,40 \mathrm{x}_{2}+266,40 \mathrm{x}_{3}-176,47 \mathrm{x}_{1} \mathrm{x}_{2}-333,07 \mathrm{x}_{1} \mathrm{x}_{3}-$ $186,32 \mathrm{x}_{2} \mathrm{x}_{3}$

$\hat{\mathrm{y}}_{\text {cúbico }}=182,87 \mathrm{x}_{1}+296,40 \mathrm{x}_{2}+266,40 \mathrm{x}_{3}-176,47 \mathrm{x}_{1} \mathrm{x}_{2}-333,07 \mathrm{x}_{1} \mathrm{x}_{3}$ $186,32 \mathrm{x}_{2} \mathrm{x}_{3}+259,61 \mathrm{x}_{1} \mathrm{x}_{3}$ 


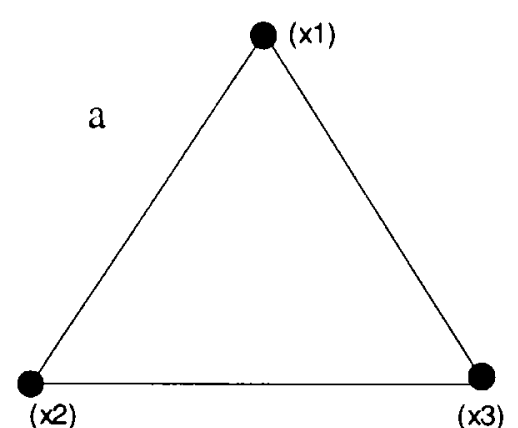

(x3)

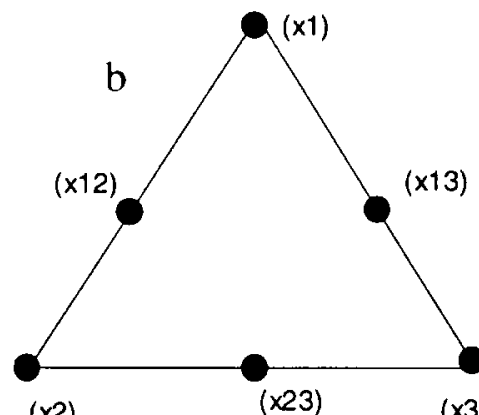

$(\times 2)$

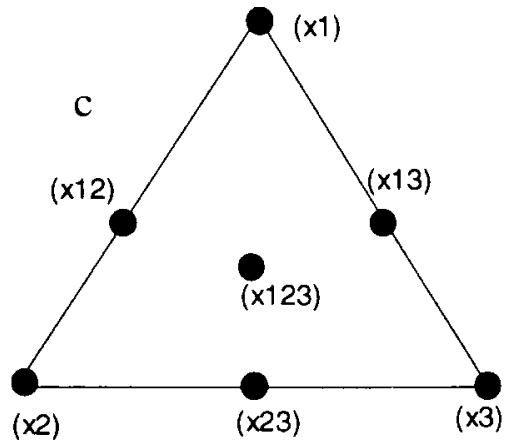

Figura 5: Distribuição de pontos da rede Simplex.

Tabela II - Comparação entre os valores observados ( $\bar{y}$ obs) e os valores previstos

\begin{tabular}{lccccc}
\hline $\mathrm{X}_{1}$ & $\mathrm{X}_{2}$ & $\mathrm{X}_{3}$ & $\begin{array}{c}\bar{y} \\
\text { (Observado) }\end{array}$ & $\begin{array}{c}\hat{y} \\
\text { respostas } \\
\text { do modelo } \\
\text { (quadrático) }\end{array}$ & $\begin{array}{c}\hat{y} \\
\text { respostas } \\
\text { do modelo }\end{array}$ \\
\hline 1 & 0 & 0 & 182,87 & 182,87 & 182,87 \\
\hline 0 & 1 & 1 & 296,20 & 296,20 & 296,20 \\
\hline 0 & 0 & 1 & 266,40 & 266,40 & 266,40 \\
\hline $1 / 2$ & $1 / 2$ & 0 & 195,00 & 195,00 & 195,00 \\
\hline $1 / 2$ & 0 & $1 / 2$ & 141,30 & 141,30 & 141,30 \\
\hline 0 & $1 / 2$ & $1 / 2$ & 231,00 & 231,00 & 231,00 \\
\hline $1 / 3$ & $1 / 3$ & $1 / 3$ & 179,40 & 171,10 & 179,40 \\
\hline $2 / 3$ & $1 / 6$ & $1 / 6$ & 161,70 & 153,85 & 153,85 \\
\hline $1 / 6$ & $2 / 3$ & $1 / 6$ & 233,80 & 222,67 & 222,67 \\
\hline $1 / 6$ & $1 / 6$ & $2 / 3$ & 223,40 & 194,79 & 194,79 \\
\hline $2 / 3$ & $1 / 3$ & 0 & 176,70 & 181,37 & 181,37 \\
\hline $2 / 3$ & 0 & $1 / 3$ & 143,50 & 136,69 & 136,69 \\
\hline $1 / 3$ & $2 / 3$ & 0 & 219,50 & 219,10 & 219,10 \\
\hline 0 & $2 / 3$ & $1 / 3$ & 280,2 & 244,74 & 244,74 \\
\hline $1 / 3$ & 0 & $2 / 3$ & 149,30 & 164,54 & 164,54 \\
\hline 0 & $1 / 3$ & $2 / 3$ & 278,50 & 234,87 & 234,87 \\
\hline $\mathrm{S}$ & 051 & & & & \\
\hline & & & & 190 & \\
\hline
\end{tabular}

Soma dos resíduos

deixados pelo modelo

(valores que não entraram $\Sigma \mathrm{e} \quad 162,05 \quad 153,76$

no calculo do modelo)

\begin{tabular}{lccc}
\hline Média dos resíduos & $\overline{\mathrm{e}}$ & 16,20 & 17,08 \\
\hline $\mathrm{R}^{2}$ & & 0,93 & 0,94 \\
\hline
\end{tabular}

\section{ANÁLISE DOS RESULTADOS E CONCLUSÃO}

Os resultados obtidos com os modelos, quando comparados com os resultados dos ensaios em amostras em função da tensão de ruptura à flexão, mostram que os modelos são significativamente efi-
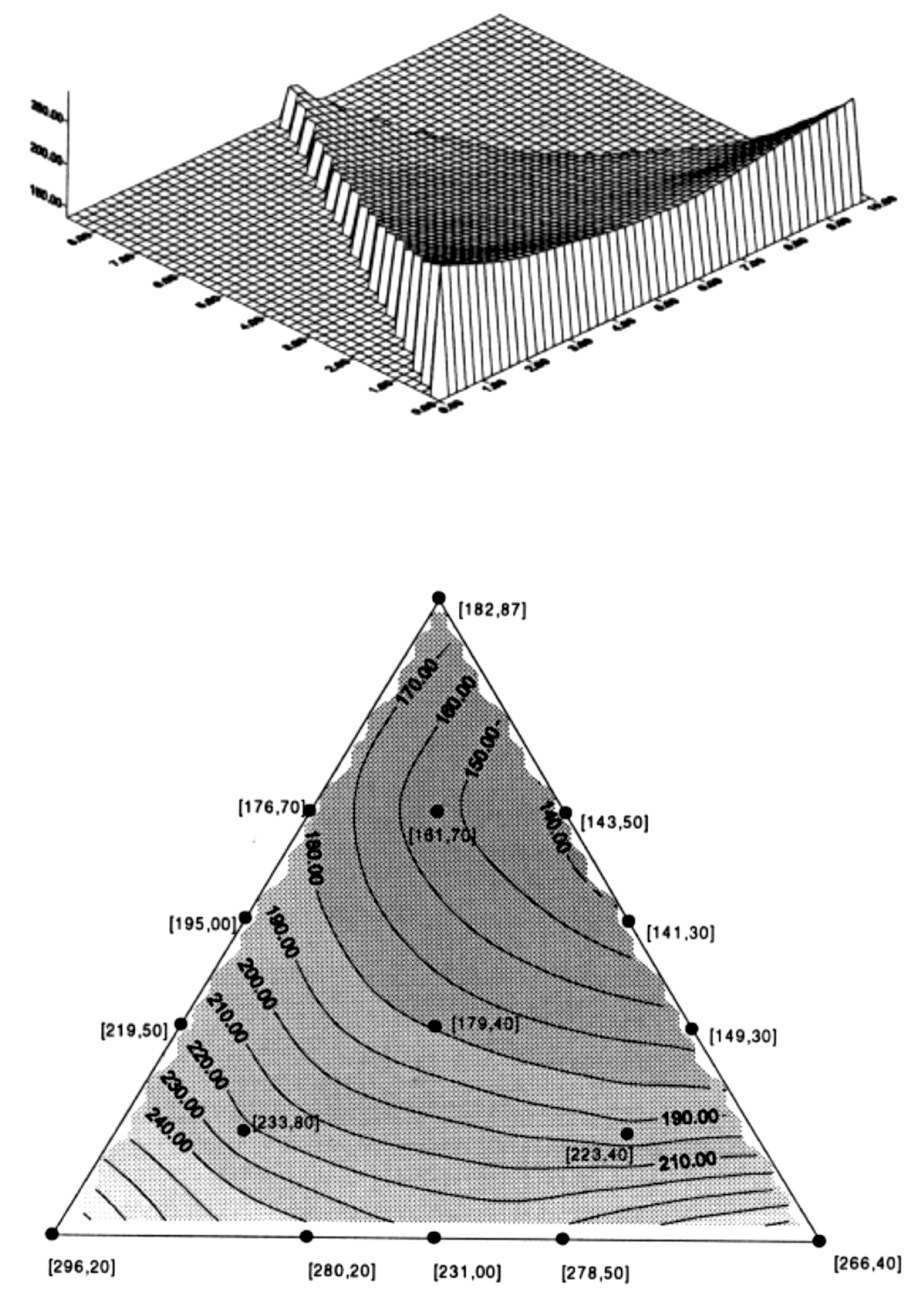

Figura 6: Superfície de respostas em curvas de nível e sua projeção para tensão de ruptura à flexão a $1200{ }^{\circ} \mathrm{C}$, calculada a partir do modelo cúbico especial.

cazes para previsão dos resultados dessa propriedade, embora o modelo linear, mais simples, mostrou pouca aderência entre resultados previstos e observados. Os resíduos, diferença entre os valores $\bar{y}$ e $\hat{y}$, de uma maneira geral são aceitáveis, visto que a matéria-prima em estudo é de origem natural e heterogênea, geralmente com alto grau de variabilidade espacial . O grau do modelo necessário para previsão dos resultados, variou do modelo linear ao 
modelo cúbico especial para o caso das amostras queimadas a temperatura de $1200{ }^{\circ} \mathrm{C}$, entretanto graus mais elevados ou mais baixos do modelo são geralmente funções das propriedades analisadas, da temperatura de queima e do grau de heterogeneidade das amostras.

Nota-se que um aumento no grau do modelo, consequentemente da complexidade e do número de ensaios requeridos, não se traduziu em uma melhor resposta para a variável ensaiada, onde o modelo quadrático foi o mais apropriado. Isto se deve ao fato de que erros sistemáticos referentes aos ensaios e a variabilidade das amostras, são do mesmo nível de significância dos coeficientes de grau mais elevados, tornando-os desprezíveis nos resultados previstos pelo modelo.

Outra importante observação a respeito da superfície de respostas, é a possibilidade de se utilizar algoritmos de otimização gráfica para a determinação da mistura adequada englobando todas as variáveis de interesse envolvidas no processo.

\section{AGRADECIMENTOS}

Os autores agradecem à Universidade Estadual do Norte Fluminense, ao técnico de laboratório André Luiz Flor Manhães e aos colegas Denise Ribeiro dos Santos e Ronaldo Lerner pelos ensaios executados.

\section{REFERÊNCIAS}

[1] J. A. Cornell, "Experiments with mixtures: designs, models and the analysis of mixture data”. 2. Ed., New York: John Wiley and Sons (1990).

[2] J. Alexandre, "Análise de matéria-prima e composições de massa utilizada em cerâmicas vermelhas". Tese de Doutorado - Campos dos Goytacazes - RJ, Universidade Estadual do Norte Fluminense - UENF (2000).

(Rec. 08/11/2000, Rev. 20/07/2001, Ac. 17/08/2001) 